\title{
La tutela de la libertad informática en la sociedad globalizada
}

\author{
Antonio- Enrique Pérez Luño \\ Universidad de Sevilla
}

Este trabajo cifra su propósito en un triple y convergente objeto: 1) En primer término, analiza algunos de los episodios más relevantes que reflejan el riesgo informático para los derechos y libertades, producidos en nuestro ámbito político-cultural. 2) En segundo término se ofrece un cuadro sumario de la respuesta jurídica a los atentados informáticos contra las libertades. Dicha respuesta se ha materializado en tres generaciones de leyes de protección de datos personales, cuyos respectivos rasgos informadores se apuntan. 3) Por último, se aboga por una respuesta jurídica global, es decir, inspirada en criterios supranacionales, para afrontar el asedio informático que subyace a determinados usos de las nuevas tecnologías en la sociedad globalizada.

\section{Informática y libertades}

En la situación tecnológica propia de la sociedad contemporánea todos los ciudadanos, desde su nacimiento, se hallan expuestos a violaciones de su intimidad perpetradas por determinados abusos de la informática y la telemática. Para responder a ese riesgo se propugna una nueva dimensión de la libertad: la libertad informática, que aparece como un nuevo derecho de autotutela de la propia identidad informática, o sea, el derecho de controlar (conocer, corregir, quitar o agregar) los datos personales inscritos en un programa electrónico.

Existe un método de probada eficacia para medir el índice de actualidad de los distintos derechos fundamentales. Me apresuro a reconocer que consiste en un método dramático: se trata de comprobar la frecuencia e intensidad con la que cada derecho es violado. Es en cualquier caso, un método acorde con la propia tematización de las libertades, que impone la reivindicación y la crítica y se halla reñido con actitudes conformistas o autocomplacientes. Por esa vía negativa, que computa agresiones en vez de evaluar los grados de satisfacción y disfrute de las libertades, se ha cumplido la premonición de un célebre voto disidente de una sentencia de 1928 de la Supreme Court norteamericana. Se afirmaba en ella que la intimidad era el derecho más apreciado por los hombres civilizados ("The right most valued by civilized men"). 
(Mr. Justice L.D. Brandeis Dissenting Opinion, en el proceso Olmstead v. United States,1928). Esa tesis ha sido corroborada por el informe británico, elaborado en 1971 por el Younger Committee on Privacy. En dicho informe se establece que la opinión pública de los países desarrollados sitúa el respeto a la vida privada en un lugar prioritario de sus aspiraciones de protección de los derechos humanos (Price of Privacy, 1971,36). Esas aspiraciones se hallan plenamente justificadas si se tiene presente que la intimidad es el derecho fundamental con mayor número de modalidades de eventual transgresión y uno de los que cuenta con mayor cantidad de víctimas reales o potenciales. Baste recordar que, en algunas investigaciones recientes sobre el asalto informático a las libertades, se han llegado a enumerar hasta cuatrocientos tipos de posibles violaciones de la privacidad. Respecto a la cantidad de sujetos pasivos de esas violaciones tenemos una experiencia próxima. El escándalo que hace algunos años agitó a nuestra opinión pública, en relación con el tráfico informatizado de datos de carácter personal, representa la confirmación de ese riesgo. Los 21 millones de ciudadanos españoles, inmediata o potencialmente, agredidos en su vida privada abren una brecha en la inconsciencia cívica y política sobre los peligros que hoy acechan al ejercicio del derecho a la intimidad. Se añade a esa magnitud cuantitativa de las violaciones contra la esfera intima su cualidad indiscriminada que, como los lances amatorios del Don Juan de Zorrilla, recorren toda la escala social desde los más altos palacios a las más humildes cabañas; de lo qu también en España hemos tenido lamentable experiencia'.

No es lícito, al menos para juristas, políticos y tecnólogos, aducir sorpresa o desconocimiento de los eventuales peligros implícitos en el uso de las nuevas tecnologías. Desde hace tres décadas, quienes han evaluado el impacto de la informática en las libertades, han alertado sobre esos peligros. Para describirlos no hay que recurrir a una retórica apocalíptica, basta la expresión estricta de un atestado.

Desde los años setenta es notorio que bancos de datos del sector público norteamericano, pertenecientes al Pentágono, la CIA o el FBI, procesan informes sobre actitudes individuales y comportamiento político que afectan a millones de ciudadanos. Datos que recabados en función de la defensa nacional o de la seguridad pública han servido, en determinadas ocasiones, para prácticas de control político y discriminación ideológica. La comunidad académica de USA sufrió una conmoción al saber que, durante la etapa de contestación estudiantil, diversas Universidades que contaban con bibliotecas informatizadas

\footnotetext{
${ }^{1}$ Vid. A.E. Pérez Luño, Nuevas tecnologias, sociedad y Derecho. El impacto socio-jurídico de las N.T. de la información, Fundesco, Madrid, 1987; id., Libertad informática y leyes de protección de datos personales, en colab. con M. Losano y M.F. Guerrero Mateus, Centro de Estudios Constitucionales, Madrid, 1989; id. La LORTAD y los derechos fundamentales, en "Derechos y Libertades", 1993, n 1, pp. 405-424; id., Manual de informática y derecho, Ariel, Barcelona, 1996, pp. 43 ss.; id., Saggi di informtica giuridica, con prólogo de V. Frosini, Giuffrè, Milano,1998, pp.147
} yss. 
proporcionaron a la policía relaciones exhaustivas de las lecturas de aquellos profesores y/o alumnos sospechosos de ser contestatarios o disidentes. Desde hace años las agencias de información comercial y de crédito nortemericano almacenan datos personales que conciernen a cientos de millones de individuos, que tras su adecuada programación, pueden trasmitirse a sus clientes en más de 10.000 aspectos diferentes (por edad, profesión, sexo, ingresos, automóvil o vivienda poseídos, pertenencia a sindicatos, partidos, o sociedades mercantiles, culturales o recreativas...) $)^{2}$.

En Francia el detonante fue un proyecto del Instituto Nacional de Estadística, por el que se pretendía atribuir a cada ciudadano un "número de identificación único" para todas sus relaciones con la administración. La fatal coincidencia, para los propugnadores del sistema, de que sus siglas respondieran a la palabra SAFARI, contribuyó a sensibilizar a partidos políticos, medios de comunicación y ciudadanos ante la amenaza de verse convertidos en las piezas a cobrar en el "safari informático". El proyecto fue finalmente suspendido y supuso una eficaz llamada de atención sobre la peligrosidad de las técnicas de over-all computer, o sea, del cruce de ficheros que permiten un control exhaustivo de la población, así como el trazado de un completo perfil de las personas $^{3}$.

Estas circunstancias pesaron en 1983 en una célebre Sentencia del Tribunal Constitucional de la República Federal de Alemania, que a instancia de los Verdes, declaró parcialmente inconstitucional la Ley del Censo de Población que obligaba a los ciudadanos germanos a suministrar datos personales para fines estadísticos. En dicha decisión jurisprudencial se reconocía el derecho a la "autodeterminación informativa", hasta entonces invocado por la doctrina jurídica, y concretado en la facultad de todo ciudadano de las sociedades democráticas de determinar: quién, qué, cuándo y con qué motivo puede conocer datos que le conciernen. La doctrina germana ha calificado a la Sentencia sobre la Ley del Censo de Población de "Sermón de la Montaña" en materia de protección de datos personales ${ }^{4}$.

En esa línea de lenguaje metafórico evangélico, entiendo que pudiera apelarse de Sentencia del "Buen Samaritano", la decisión de nuestro Tribunal Constitucional 254/1993 de 20 de Julio. En ella el TC reconoce y ampara el derecho de los ciudadanos a conocer los datos personales que les conciernen y se hallan registrados en archivos informatizados administrativos. Esta decisión tuvo como antecedentes las Sentencias de la Audiencia Territorial de Pamplona

${ }^{2}$ Cfr. R. T. Nimmer, The Law of Computer Technology, Warren, Gorhan \& Lamont, New York, 1985 , pp. 81 y ss.

${ }^{3}$ Cfr. el vol. col. Emergence du droit de l'informatique, Editions des Parques, París, 1983.

${ }^{4}$ Vid., E. Denninger, El derecho a la autodeterminación informativa, trad. cast. de A.E. Pérez Luño, en el vol. col. Problemas actuales de la documentación y la informática juridica (Actas del Coloquio Internacional celebrado en la Universidad de Sevilla, 5 y 6 de marzo de 1986), a cargo de A.E. Pérez Luño, Tecnos \& Fundación Cultural Enrique Luño Peña, Madrid, 1987. 
(Sala de lo Contencioso- Administrativo) de 7 de Febrero de 1989 y del Tribunal Supremo (Sala Tercera) de 30 de Abril de 1990, que habían desestimado el acceso de un ciudadano a sus datos personales incluidos en archivos automatizados de la Administración del Estado, alegando la falta de desarrollo legislativo del Convenio europeo de protección de datos personales. Frente a esas sucesivas denegaciones del acceso a los datos personales, basadas en una motivación que metafóricamente pudiera calificarse de "farisáica" y "levítica" el TC actuó en este supuesto como un "Buen Samaritano". La del TC se mueve en un doble plano argumentativo:

1) De un lado, acoge la dimensión positiva del derecho a la intimidad como facultad de control sobre los datos relativos a la propia persona. Acepta también expresamente, por vez primera, las nociones de "libertad informática" y habeas data, como integrantes de la garantía de la intimidad frente a la informática consagrada en el art. 18.4 CE (FJ 7). Estos conceptos y categorías han sido ampliamente utilizados por la doctrina y jurisprudencia extranjeras en materia de protección de datos personales y estimo que me incumbe una cierta responsabilidad en su difusión en nuestra experiencia jurídica.

2) El otro aspecto nuclear de ese fallo reside en la reafirmación por parte del TC de su doctrina tendente a reconocer la aplicación inmediata de los derechos fundamentales, en este caso de la libertad informática que dimana del art. 18.4 CE, sin que sea necesaria una interpositio legislatoris, es decir, un desarrollo legislativo, para su plena eficacia (FJ 6). El TC estima que el cumplimiento o no por los poderes públicos de los tratados internacionales, en particular del Convenio europeo de protección de datos personales de 1981, es independiente para asegurar la protección de los derechos fundamentales establecida en el art. 53.3 de la CE (FJ 5). De lo que implícitamente se infiere que el TC no cuestiona la plena incorporación del Convenio europeo al ordenamiento jurídico español, en virtud del art.96.1 de la CE, y su plena eficacia; a lo que, por otra parte, también sería de aplicación su propia jurisprudencia impugnatoria de la interpositio legislatoris en materia de derechos fundamentales. Tesis que se corrobora por la expresa llamada del TC, apoyándose en el art. 10.2 CE, al Convenio como cauce para configurar el sentido y alcance de la libertad informática consagrada en el art. 18.4 de la $\mathrm{CE}$. En diversas publicaciones de estos últimos años he venido insistiendo en la eficacia inmediata del art. 18.4 CE, así como en la del Convenio europeo, por lo que debo congratularme de que este planteamiento haya hallado acogida en esta decisión del TC ${ }^{5}$.

No huelga advertir que en nuestro propio país existían también antecedentes : inquietantes de abusos informáticos en las libertades. Desde hace tiempo las

${ }^{5}$ Vid. A.E. Pérez Luño, Libertad informática y leyes de protección de datos personales, cit., pp. 163 ss.; La incorporación del Convenio Europeo sobre protección de datos personales al ordenamiento jurídico español, en el $\mathrm{n}^{\circ} 17$ monográfico de ICADE. Revista de las Facultades de Derecho y Ciencias Económicas y Empresariales, sobre Informática y Derecho, 1989, pp. 27 ss.; id., Panorama general de la legislación española sobre protección de datos, en el vol. col. Implicaciones socio-juridicas 
instituciones y personas más receptivas a los riesgos de contaminación informática de las libertades han mostrado su preocupación por la persistencia en archivos policiales, ahora informatizados, de datos relativos a actividades políticas, o conductas "desviadas" realizadas en el régimen anterior; el "caso Curiel" constituye un elocuente testimonio. También se han presentado quejas ante el Defensor del Pueblo por la transmisión incontrolada de historias clínicas, así como de datos sobre actitudes y circunstancias personales de funcionarios y trabajadores, elaboración de bases de datos incontroladas sobre la solvencia patrimonial y crédito (los célebres censos de morosos e insolventes) ...

La injerencia del ordenador en las diversas esferas y en el tejido de relaciones que conforman la vida cotidiana se hace cada vez más extendida, más difusa, más implacable. Por ello resulta cada vez más apremiante el reconocimiento del derecho a la libertad informática

\section{Evolución de los sistemas de tutela de la libertad informática: las "generaciones de leyes de protección de datos"}

Si una de las notas distintivas de los sistemas jurídicos actuales es la de su acelerada e incesante mutación, este rasgo se hace manifiesto con especial intensidad en un sector como el de las relaciones entre la Informática y el Derecho, en el que, constantemente, cada Feria tecnológica abre nuevas proyecciones informáticas al Derecho, o innova bienes informáticos que requieren nuevos procedimientos de tutela jurídica, o da a conocer dispositivos que condenan al anacronismo los medios de protección jurídica anteriormente existentes. Para comprender mejor lo que suponen los avances informáticos, que están operando una auténtica revolución en los sistemas de documentación jurídica, conviene reparar en lo que han supuesto las propias transformaciones tecnológicas. Es sabido que el progreso de la electrónica registra la sucesión de cuatro generaciones de ordenadores. La primera se considera que aparece con el ENIAC (Electronic Numerical Integrator and Calculator), que fue el primer computador totalmente electrónico, diseñado en 1946 por John Mauchly y Prosper Eckert y funcionaba mediante 18.000 tubos o válvulas electrónicas. En esta etapa y cinco años más tarde salió al mercado el primer ordenador comercial: el UNIVAC 1, construido por la firma IBM. La segunda generación de ordenadores, que ya usaban transistores, se desarrolló a partir de 1958. Los ordenadores de la tercera generación, que empleaban circuitos integrados, se dẹsarrolla entre los años 1965 a 1976. La cuarta generación se caracterizó por la aparición de los microprocesadores y por la técnica de integración de circuitos a gran escala (VLSI, Very Large Scala Integration). En la actualidad se alude a una quinta generación de ordenadores, para denotar las últimas

de las tecnologías de la información. Encuentro 1991, Fundación Citema, Madrid, 1992, pp. 25 y ss. 
investigaciones sobre computadoras inteligentes simuladoras del comportamiento humano en la formulación de decisiones, solución de problemas y otras actividades de carácter lógico.

A esa evolución generacional de los avances tecnológicos le ha correspondido una evolución también generacional de las libertades. La mutación histórica de los derechos humanos ha determinado la aparición de sucesivas "generaciones" de derechos. Los derechos humanos como categorías históricas, que tan sólo pueden predicarse con sentido en contextos temporalmente determinados, nacen con la modernidad en el seno de la atmósfera iluminista que inspiró las revoluciones burguesas del siglo XVIII. Ese contexto genético confiere a los derechos humanos unos perfiles ideológicos definidos. Los derechos humanos nacen, como es notorio, con marcada impronta individualista, como libertades individuales que configuran la primera fase o generación de los derechos humanos. Dicha matriz ideológica individualista sufrirá un amplio proceso de erosión e impugnación en las luchas sociales del siglo XIX. Esos movimientos reivindicativos evidenciarán la necesidad de completar el catálogo de los derechos y libertades de la primera generación con una segunda generación de derechos: los derechos económicos, sociales y culturales. Estos derechos alcanzarán su paulatina consagración jurídica y política en la sustitución del Estado liberal de Derecho por el Estado social de Derecho.

La estrategia reivindicativa de los derechos humanos se presenta hoy con rasgos inequivocamente novedosos al polarizarse en torno a temas tales como como el derecho a la paz, los derechos de los consumidores, el derecho a la calidad de vida, o la libertad informática. En base a ello, se abre paso, con intensidad creciente, la convicción de que nos hallamos ante una tercera generación de derechos humanos complementadora de las fases anteriores, referidas a las libertades de signo individual y a los derechos económicos, sociales y culturales. De este modo, los derechos y libertades de la tercera generación se presentan como una respuesta al fenómeno de la denominada " contaminación de las libertades" (liberties' pollution), término con el que algunos sectores de la teoría social anglosajona aluden a la erosión y degradación que aqueja a los derechos fundamentales ante determinados usos de las nuevas tecnologías ${ }^{6}$.

Si la informática y las libertades han evolucionado generacionalmente nada tiene de particular que el punto de encuentro entre ambas categorias, es decir, la libertad informática responda, a su vez, a una decantación generacional.En efecto, la experiencia legislativa de estos últimos años registra una sucesiva decantación desde las leyes de la primera generación, basadas en la autorización previa de los bancos de datos en una etapa en la que los equipos informáticos

\footnotetext{
${ }^{6}$ Vid. A.E. Pérez Luño, Le generazioni dei diritti umani, en el vol. col. a cargo de F. Riccobono, Nuovi diritti dell'età tecnologica, (Atti del Convegno tenuto a Roma presso la Libera Università Internazionale degli Studi Sociali, 5 e 6 maggio 1989), Giuffrè, Milano,1991, pp. 139 ss.; Las generaciones de derechos fundamentales, en "Revista del Centro de Estudios Constitucionales", 1991, n. 10, pp. 203 y ss.
} 
eran escasos, voluminosos y fácilmente localizables; a las leyes de la segunda generación, cuyo principal objetivo fue la garantía de los datos "sensibles", por su inmediata incidencia en la privacidad o su riesgo para prácticas discriminatorias; y, en la actualidad, a las de la tercera generación, que se han hecho cargo de la revolución microinformática con la consiguiente difusión capilar de los bancos de datos. Ello ha hecho prácticamente inviable el control previo de los equipos informáticos, sobre el que operaron las normas de la primera generación; al tiempo que la tutela de las informaciones ya no puede quedar circunscrita al factor estático de su calidad, según el criterio predominante en la segunda generación de leyes de protección de datos, sino que debe hacerse extensiva a la dinámica de su uso o funcionalidad.

En nuestro ordenamiento jurídico, la tutela de la libertad frente a los abusos informáticos reviste rango constitucional, ya que se halla prevista en el art. 18.4 CE. para desarrollar ese precepto de la Constitución se promulgó la Ley orgánica 5/1992 de 29 de octubre, de regulación del tratamiento automatizado de los datos de carácter personal (LORTAD). La LORTAD ha sido recientemente derogada por la Ley Orgánica 15/1999, de 13 de diciembre de protección de datos personales. Ambos textos parecen querer optar por el modelo de las denominadas "Leyes de protección de datos de la tercera generación". La Exposición de Motivos de la LORTAD concebía la protección de los bancos de datos personales desde una perspectiva funcional; no se limitaba a su tutela en cuanto meros depósitos de informaciones, "sino también, y sobre todo, como una globalidad de procesos o aplicaciones informáticas que se llevan a cabo con lo datos almacenados y que son susceptibles, si llegasen a conectarse entre sí, de configurar el perfil personal". Dicho perfil era considerado, en la propia Exposición de Motivos, como la reputación o fama que es expresión del honor y que "puede ser valorado, favorable o desfavorablemente, para las más diversas actividades públicas o privadas, como pueden ser la obtención de un empleo, la concesión de un préstamo o la admisión en determinados colectivos". La LORTAD se propuso por tanto, tutelar la calidad de los datos, pero no en sí mismos, sino en función de evitar que su informatización permita o propicie actividades discriminatorias. Este mismo propósito persigue el art. 13 de la vigente Ley 15/99 de protección de datos personales. Asimismo reforzaba el modelo de tutela dinámica el protagonismo que reviste la actuación de Agencia de protección de datos, que asume las principales competencias en materia de la organización de los mecanismos de tutela ${ }^{7}$.

\footnotetext{
${ }^{7}$ Cfr.: Cascajo Castro, J.L, Tratamiento automatizado de los datos de carácter personal, en el vol.col. a cargo de J.M. Sauca, Problemas actuales de los derechos fundamentales, Universidad Carlos III de Madrid \& Boletín Oficial del Estado, Madrid, 1994, p. 363 ss.; Lucas Murillo de la Cueva, P. Informática y protección de datos personales. Estudio sobre la Ley Orgánica 5/1992, de Regulación de Tratamiento Automatizado de los Datos de Carácter Personal, Centro de Estudios Constitucionales, Madrid ,1993, pp. 74 ss.; Martín Casallo, J.J., Agencia de Protección de Datos. qué es y qué finalidad persigue, en "Actualidad Informática Aranzadi", $\mathrm{n}^{0}$ 13, 1994, pp. 1-2.
} 
La Directiva 95/46/CE se inscribe también en el ámbito de la leyes de la tercera generación. En este texto se da una importancia decisiva a los "tratamientos" de los datos personales, ante la importancia de las técnicas de transmisión de dichos datos en el marco de la sociedad de la información (Considerandos, 14 y 26). Por ello la Directiva reconoce expresamente el derecho de las personas " a no verse sometidas a una decisión con efectos jurídicos sobre ellas o que las afecte de manera significativa, que se base únicamente en un tratamiento automatizado de datos destinados a evaluar determinados aspectos de su personalidad, como su rendimiento laboral, crédito, fiabilidad, conducta, etc." (art. 15). La Directiva denomina a estas prácticas "decisiones individuales automatizadas", que corresponde a lo que la LORTAD califica de "perfiles de personalidad". Al propio tiempo, la importancia que la Directiva otorga a la Autoridad de control y al Grupo de protección refuerzan su opción en favor de un sistema de tutela dinámica de los datos personales.

\section{Conclusión: La tutela de la libertad informática bajo el signo de la globalización}

Los problemas actuales referidos a la protección de la libertad informática deben ser estudiados desde una perspectiva de totalidad. La sociedad humana es multidimensional y, asimismo, lo son sus problemas éticos, jurídicos y políticos. Por eso, hay que captar la dinámica y compleja red de sus conexiones globales. La tendencia hacia la globalización viene impuesta por el carácter interdependiente, multicéntrico y multicultural de los fenómenos que gravitan sobre la tulela de las libertades. Resulta clarificadora la idea aportada en un documento de la conferencia del Club de Roma celebrada en Santander en 1985: "comprenderíamos mejor la dinámica de la interdependencia si no imagináramos el mundo como un mapa de naciones, sino como un mapa meteorológico, en el que los frentes climáticos se arremolinan y se desplazan con independencia de las fronteras nacionales y los frentes de bajas y altas presiones crean condiciones meteorológicas a gran distancia" 8 .

Esa interdependencia ha producido una paulatina erosión del poder de los Estados para controlar sus problemas y alcanzar sus objetivos, que hoy tienen una dimensión global, que tan sólo puede enfocarse y solucionarse en términos de cooperación internacional. Esta exigencia incide, de forma muy especial, en la tutela de la libertad informática, pues resulta evidente que las agresiones a este derecho poseen una dimensión internacional y, por ello, su

- respuesta no puede quedar entorpecida por las limitaciones propias de los ordenamientos jurídicos nacionales.

Concebir la libertad informática desde una perspectiva globalizadora, implica avanzar hacia soluciones jurídicas supranacionales. El constitucionalismo

\footnotetext{
${ }^{8}$ Vid. A.E. Pérez Luño, Manual de Informática y derecho, cit., pp. 59 y ss.
} 
actual registra tendencias que le otorgan una peculiar fisonomía. En el seno de estas orientaciones quizás sean las más significativas las que conciernen a los procesos de comunicación, recepción y unificación constitucional. Entre ellos destacan los actuales empeños dirigidos a la elaboración de un Derecho Constitucional Común Europeo. En aparente paradoja el término "Derecho Constitucional Común Europeo (DCCE)", supone una expresión nueva de vieja raigambre jurídica. Se trata de una expresión nueva en cuanto ha sido acuñada en fecha reciente por el Profesor Peter Häberle, Catedrático de Filosofía del Derecho y Derecho Público en las Universidades de Bayreuth y St. Gallen, pero su modelo inspirador se remonta al ius commune. El DCCE se halla integrado por " un conjunto de principios constitucionales «particulares» que resultan "comunes» a los diferentes Estados nacionales europeos, tanto si han sido positivados como si no". Dichos "principios comunes" proceden de las constituciones de los Estados de Derecho europeos, del Derecho constitucional consuetudinario de esos Estados, así como del "Derecho europeo" surgido de la Comunidad Europea, del Consejo de Europa y de la Conferencia para la Seguridad y la Cooperación en Europa. Esos principios integradores del DCCE están destinados a cumplir en el ámbito del Derecho público un papel análogo al desempeñado por el ius commune como fundamento de las instituciones del Derecho privado; conformándose como un auténtico ius commune constitutionale ${ }^{9}$.

Al DCCE se le asigna como objetivo o fin prioritario el contribuir a forjar una Constitución común para Europa. En reiteradas ocasiones, con referencia expresa a la CEE, se ha denunciado que se trata de una organización institucional económica y política que carece de Constitución. El DCCE vendría a colmar esa carencia e incluso a rebasarla, ya que la auspiciada Constitución europea lo sería de toda la Europa cultural y no sólo de la comunitaria. La Constitución europea representaría la casa constitucional común europea (das gemeinsame europäische Verfassungshaus), con múltiples alcobas hacia el interior y el exterior, pero con autoconsciencia de que sus cimientos son comunes. La Constitución común europea implicaría la culminación del método comparativo y el fundamento cultural del DCCE. Sería el producto de la estrecha y profunda colaboración, por vía comparativa, de las distintas culturas europeas $^{10}$.

En el plano de la defensa de la libertad informática, la Ley Orgánica 15/99 y la Directiva representan eslabones de esa cadena jurídica conformadora del sistema constitucional europeo de las libertades. La constribución de estos textos a tutelar los datos personales se enmarca en el proceso conformador del Derecho Constitucional Común Europeo, en uno de sus ámbitos mas nove-

\footnotetext{
${ }^{9}$ P. Häberle, Derecho Constitucional Común Europeo, trad. cast. de E. Mikunda, en " Revista de Estudios Políticos", 1993, n. 79, p. 11.

${ }^{10}$ Vid. A.E. Pérez Luño, El Derecho Constitucional Común Europeo:apostillas en torno a la concepción de Peter Häberle, en "Revista de Estudios Políticos", n 88, pp. 165 y ss.
} 
dosos y que suscita una creciente sensibilidad cívica. Por más que sabido nunca es inoportuno insistir en que la eficacia de la tutela de las libertades y, por eso, también de la libertad informática, depende tanto de la calidad técnica de los cauces previstos en las normas constitucionales y las leyes que la desarrollan, como de la convicción de los operadores jurídicos y los ciudadanos por hacer de esas normas una experiencia tangible en la vida cotidiana de las sociedades democráticas ${ }^{11}$.

${ }^{11}$ Cfr. A.E. Pérez Luño, Derechos humanos, Estado de Derecho y Constitución, Tecnos, Madrid, $6^{\text {a }}$ ed., 1999 , pp. 345 y ss. 